\title{
Mechanism for Redox Exfoliation of Layered Transition Metal Dichalcogenides
}

Ali Jawaid, Allyson Ritter, Richard Vaia

Materials and Manufacturing Directorate, Air Force Research Laboratory,

Wright-Patterson AFB, Ohio 45433-7702

*richard.vaia@us.af.mil, Phone number 937-785-9209

\section{Table of Contents:}

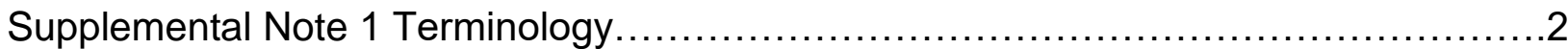

Figure S1 Surface Cleaning of Various Sources of MoS2 Powders $\ldots \ldots \ldots \ldots \ldots \ldots \ldots \ldots . \ldots$

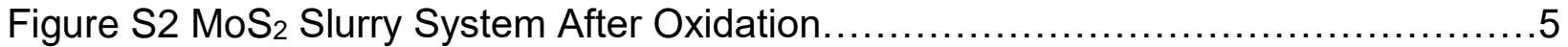

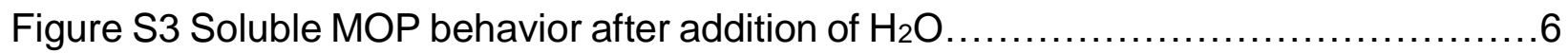

Figure S4 Optical Images of Oxidation In Different Media \& Oxidants.....................

Figure S5 Ex-situ POM Assembly Characterization....................................

Figure S6 Qualitative Summary of Control Redox Exfoliations..........................

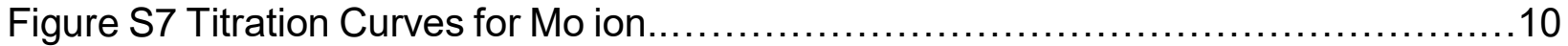

Figure S8 Tauc Plots of $\mathrm{MoS}_{2}$ dispersions ..............................................

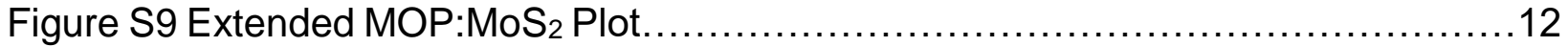

Figure S10 Addition of Shear Force To Oxidized Slurries.............................13

Figure S11 Kinetic Plot of Mo ion Depletion........................................... 14

Figure S12 Line Cuts of Exfoliated Flakes..........................................

Figure S13. Lateral Size of Exfoliated Flakes.......................................... 16

Figure S14 Recycled Exfoliation Reactions......................................... 17

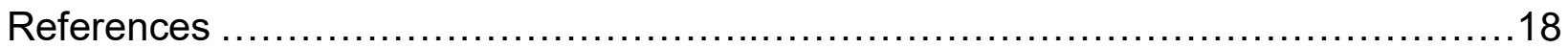


Supplemental Note 1: Terminology

To avoid confusion across disciplines and communities, we have utilized the following terminology to be as accurate and descriptive as possible. As with many layered inorganic materials, the as-received LTMD powder is comprised of a broad heterogeneous collection of individual particles that consist of an agglomeration of crystallites (or primary particles). The lateral size of these crystallites establishes the maximum lateral size of few-to-monolayer product and is equal-to, or less than, the size of the as-received crystallite powder size. Typically, the maximum lateral size of few-tomonolayer LTMDs is not achieved due to crystallite scission and fracturing. The size of these agglomerates, termed the powder size, reflects the aggregation of primary crystallites. Note that the powder sizes are not measurements of the primary crystallite size (or crystallite grain size).

Solutions compromising LTMDs are referred to as a slurry: this contains all components of the liquid media, reactants, and products. The LTMD colloidal dispersion is the slurry after purification to remove excess reactants, un-delaminated LTMD powder, and undesired by-products. It nominally contains dispersed (colloidally-stable) and delaminated LTMDs (flakes) with a broad distribution of lateral size and thickness. Subsequent size selection of these flakes leads to dispersions with enriched subpopulation of few-to-monolayer, and even monolayer, product. We will refer to these subpopulations as exfoliated LTMDs. The quality of the exfoliated LTMDs can be measured spectroscopically (e.g. A-exciton), as well as morphologically (distribution of layer width and thickness). As the thickness distribution of the dispersed ensemble skews toward few-to-monolayer enrichment, the A-exciton red-shifts in extinction 
measurements, towards the optical monolayer gap $(1.85 \mathrm{eV} ; \sim 668 \mathrm{~nm})$. Exfoliation will refer to the complete reaction and work-up procedure leading to the exfoliated LTMDs, whereas delamination will refer to the mechanism in which the crystallite is broken apart (e.g. sequential separation of layers from the crystallite v. swelling of the crystallite). Reaction yield of a specific product (e.g. colloidal dispersion or few-to-monolayer exfoliated LTMDs) and production rate is defined with respect to the mass of as-received LTMD powder and the concentration of the initial reactants (e.g. as-received LTMD powder, reactants, etc.), respectively. 
A

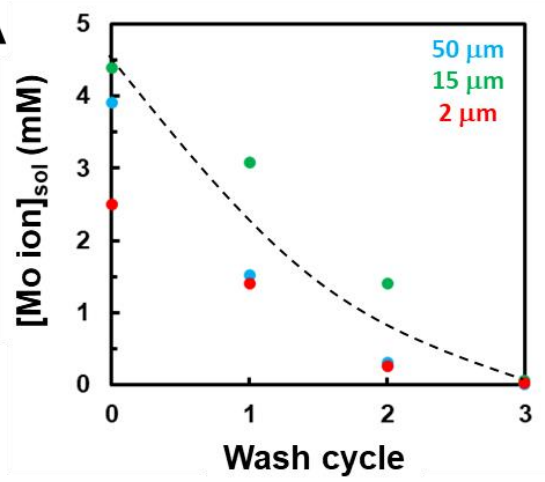

B

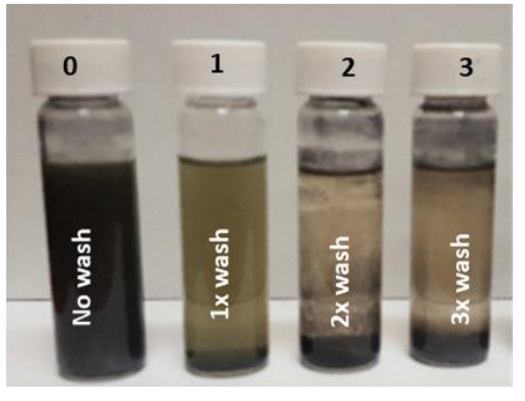

Table S1

\begin{tabular}{lccc}
\hline MoS $_{2}$ Source & $\begin{array}{c}\text { mg impurity I } \\
\text { 25g MoS }\end{array}$ & $\begin{array}{c}\text { \%surface } \\
\text { impurity }\end{array}$ & Lot \# \\
\hline Natural $\mathrm{MoS}_{2}$ & 580 & $2.32 \%$ & N/A \\
Sigma (2 micron) & 504 & $2.01 \%$ & MXCD2686 \\
Sigma (6 micron) & 325 & $1.30 \%$ & WXBC8313V \\
Materion (15 micron) & 355 & $1.42 \%$ & LC-I-89 \\
Alfa-Aesar (50 micron) & 100 & $0.40 \%$ & R06F027 \\
\hline
\end{tabular}

Figure S1. Removal of Surface Impurities From MoS $_{2}$ Powders. Refluxing powders in ethanol/acetone solvent media liberates surface bound corrosion products such as molybdates, sulfates, and polyoxomolybdates. The amount of these impurities varies significantly between supplier, where upwards of $2+\%$ of oxidation products are observed for smaller grains. All powders received were washed (see methods) prior to any exfoliation reactions were carried out.

(A) Multiple wash cycles liberate surface molybdates as indicated by decreasing $\mathrm{Mo}^{\mathrm{VI}}$ ions after sequential wash cycles. (B) When $\mathrm{MoS}_{2}$ is subjected to redox exfoliation, decreasing amounts of flakes are exfoliated, indicating a correlation between surface bound impurities and ability to exfoliate as received flakes. After c.a. 3 washes, the concentration of Mo ions in the liquor is not observable via the thiocyanate assay. This indicates that as surface cleaning is performed, additional oxidative products are not formed while simultaneously allowing for extraction and purification of bulk powders received. While other groups have reported alternative cleaning routes using aqueous dispersions,${ }^{1}$ it has not been established that these procedures remove all corrosion products while simultaneously suppressing additional oxidative events.

Table S1: Mass of impurities extract from washing raw $\mathrm{MoS}_{2}$ powders. The impurities are typically deep blue, and likely contain molybdates, sulfates, and mixed POM species. 


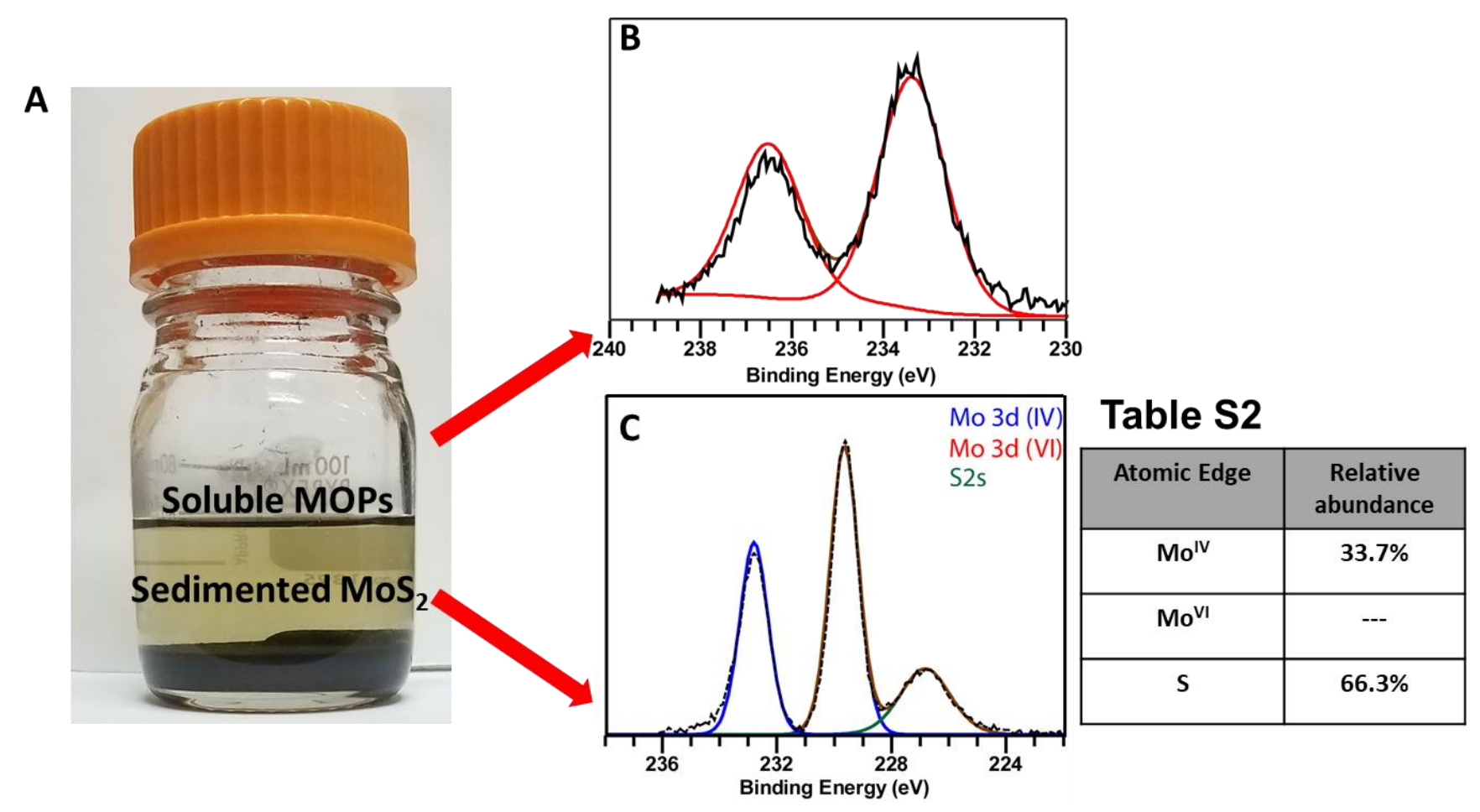

Figure S2 Redox Exfoliation after Oxidation. A) After oxidation, the $\mathrm{MoS}_{2}$ separates from soluble MOPs if allowed to stand (c.a. 5 minutes). XPS Spectra of each component in the system (i.e. sedimented $\mathrm{MoS}_{2}$ and soluble MOPs) indicate the soluble MOPs contain only $\mathrm{Mo}^{\mathrm{VI}}$ species (B) and the solid sediment only contains $\mathrm{MoS}_{2}(\mathrm{C})$ species, as previously observed. ${ }^{2}$ The preservation of the $\mathrm{MoS}_{2}$ compositional stoichiometry indicates the oxidation does not corrode the basal surface of the LTMD and the liberated MOPs are likely derived from edge sites. The ability to separate solution soluble MOPs from $\mathrm{MoS}_{2}$ allow for quantification of both extent of oxidation as well as amount of soluble Mo ions present as oxidation, MOP assembly, and surface adsorption occurs.

Table S2. Atomic composition of sediment indicating expected S:Mo ratio of 2:1 


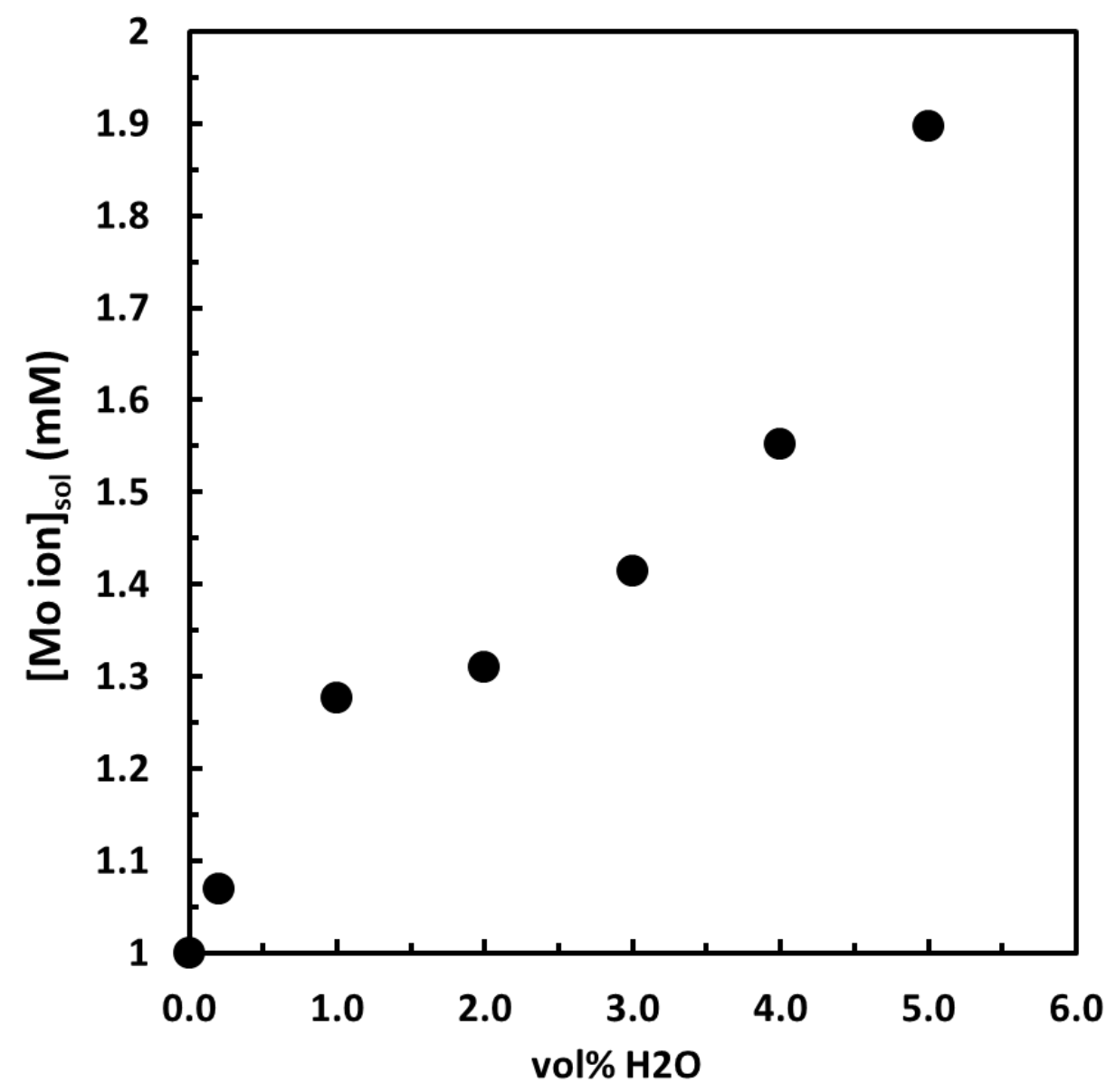

Figure S3. Effect of $\mathrm{H}_{2} \mathrm{O}$ on [MOP] eq. After equilibration of $\mathrm{MoS}_{2}$ oxidized with $\mathrm{CHP}$ (30 $\mathrm{mg} / \mathrm{mL} ; 0.2: 1 \mathrm{CHP}: \mathrm{MoS}_{2} \mathrm{~mol} / \mathrm{mol}$ ), addition of $\mathrm{H}_{2} \mathrm{O}$ to the slurry results in a dramatic increase in soluble MOPs. This increase is either due to secondary oxidation events due to introduction of $\mathrm{H}_{2} \mathrm{O}$ or displacement of MOPs adsorbed to the $\mathrm{MoS}_{2}$ surface. Due to the large increase in the concentration of Mo ions, we believe the $\mathrm{H}_{2} \mathrm{O}$ acts as a secondary oxidant, as the surface equilibration of MOPs on the $\mathrm{MoS}_{2}$ surface cannot account for the large increase in the soluble Mo ion concentration. Thus, to avoid complications in quantification in all subsequent experiments, care was taken to ensure anhydrous reaction environments. 
A

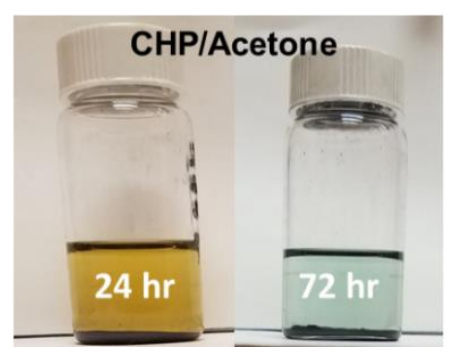

B

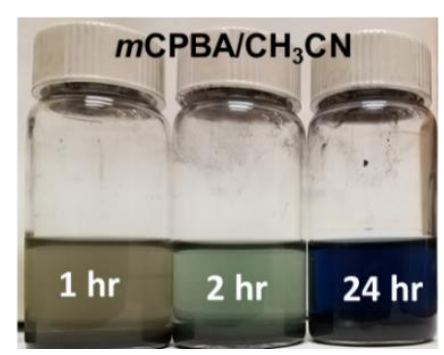

C

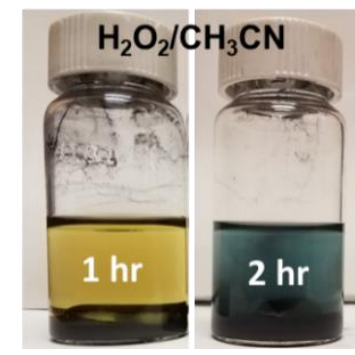

Figure S4. Oxidation Of $\mathrm{MoS}_{2}$ in different solvents / oxidants. A) In CHP/Acetone mixtures, initially MOPs are formed and slowly evolve into POMs within 48 hours. B) Similarly, in $m \mathrm{CPBA} / \mathrm{CH}_{3} \mathrm{CN}$ slurries, oxidation results in immediate POM formation. C) Similar to $m$ CPBA, oxidation results in immediate POM assembly. Both $m C P B A$ and $\mathrm{H}_{2} \mathrm{O}_{2}$ challenge reagent level control of MOP generation and POM assembly.

Note, while it is possible to exfoliate $\mathrm{MoS}_{2}$ in these formulations, correlation of MOPs, POMs, and delaminated $\mathrm{MoS}_{2}$ proved to be difficult. The transient nature of MOPs and POMs in these media resulted in the inability to efficiently track MOP to POM conversion, despite the overall mechanism proceeding as described in the main text. For instance, stirring a solution of $\mathrm{MoS}_{2}$ treated with $\mathrm{H}_{2} \mathrm{O}_{2}$ in acetonitrile results in an initially highly yellow colored solution, which subsequently transforms to an emerald colored solution, and finally a deep blue solution. continual stirring of this solution affords colloidally stable $\mathrm{MoS}_{2}$ without introduction of a reductant, as the stabilizing POMs (blue color) form spontaneously. The spontaneous formation of these highly colored species requires partial reduction of $\mathrm{Mo}^{\mathrm{VI}}$ to $\mathrm{Mo}^{\mathrm{V}}$. We believe that the LTMD surface acts as a redox active species due to the fact that if the soluble MOPs are separated from the MoS 2 , no POMs visibly form (solution remains yellow). However, if continually stirred in the presence of $\mathrm{MoS}_{2}$, a blue color develops rapidly, indicating electron transfer to the POM macroanions 

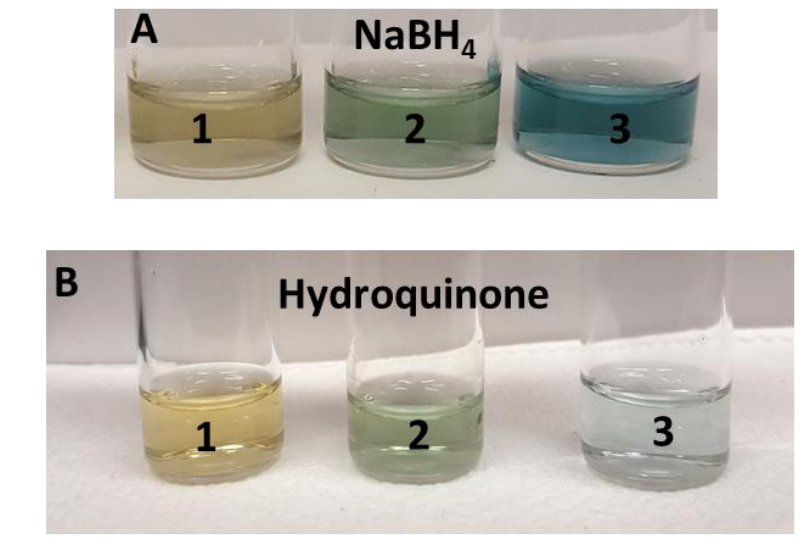

Table S3

\begin{tabular}{|c|c|}
\hline Treatment & [Mo ion] (mM) \\
\hline 1. Oxidized & 0.85 \\
\hline 2. Reduced (green) & 0.84 \\
\hline 3. Reduced (blue) & 0.81 \\
\hline
\end{tabular}
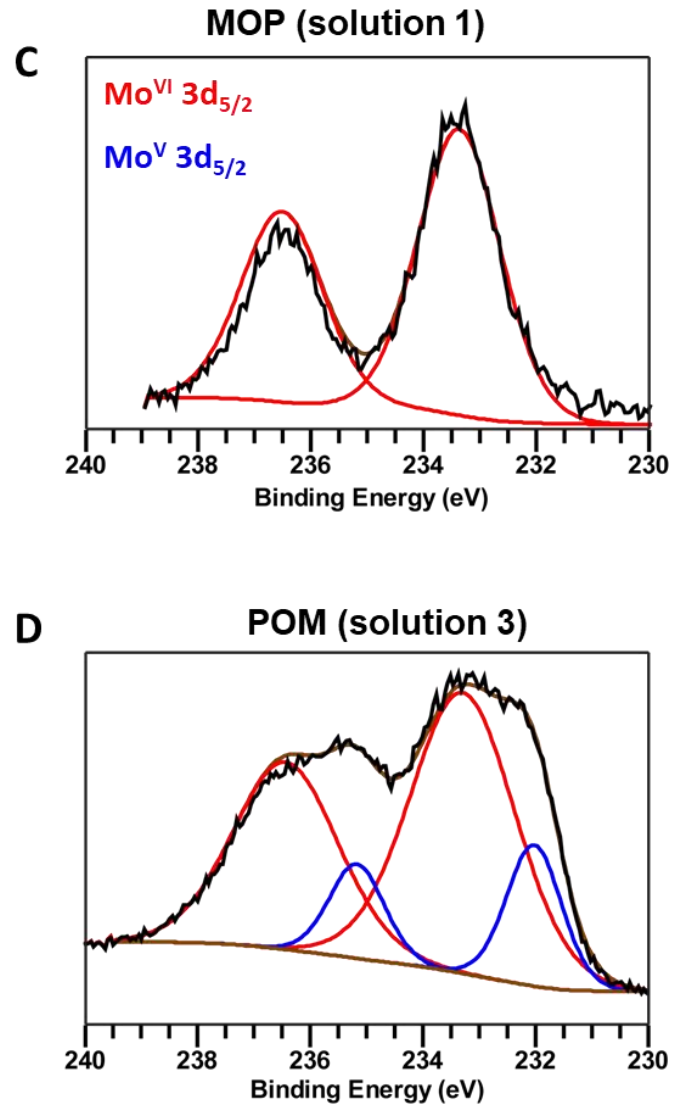

Figure S5. Digital images of oxidized supernatants and POM formation after addition of A) $\mathrm{NaBH}_{4}$ and B) Hydroquinone. Testing these supernatants for Mo ions results in a positive test indicating the POMs formed in solution are dynamic and can be disassembled and complex with the thiocyanate ion. The tabulated data are after reduction with hydroquinone. C) XPS Spectra of MOPs (solution 1) and D) after reductant is added (hydroquinone, solution 3). The emergence of $\mathrm{Mo}^{\vee}$ species is indicative of mixed valent, charged POM assemblies in solution.

Table S3: thiocyanate assays of each solution indicates the absolute amount of soluble Mo ions does not change upon reductive treatment of MOPs. 


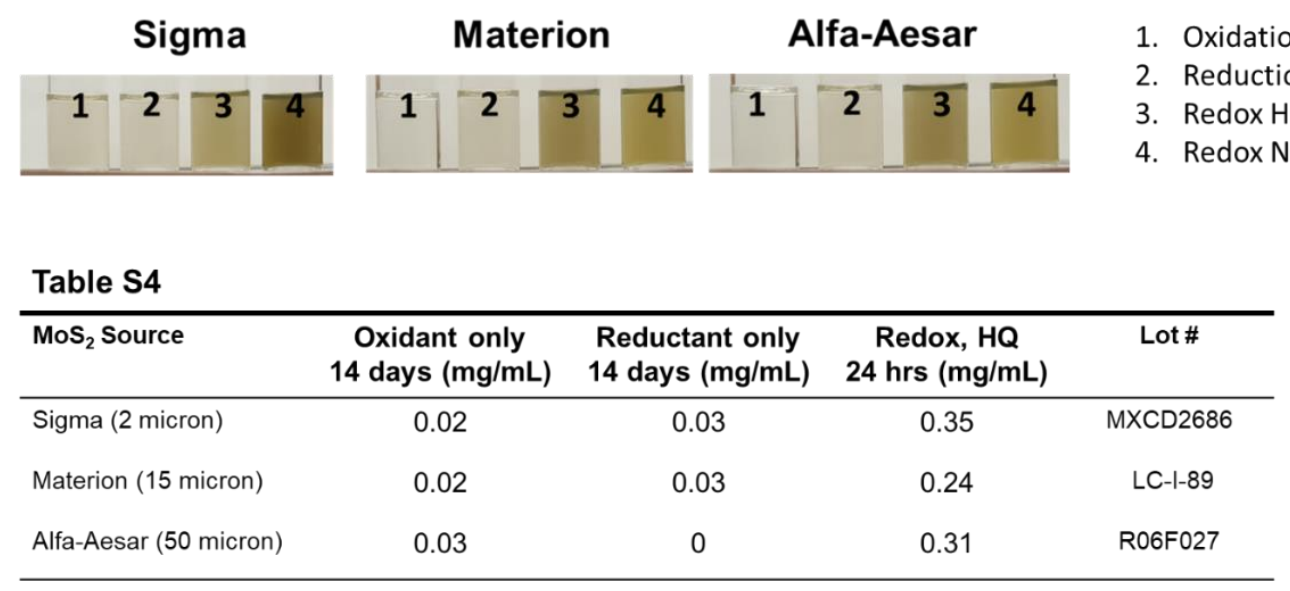

Figure S6. Redox Exfoliation After Addition Of Reductant. All reactions carried out under ambient conditions under gentle stirring via benchtop stirplate. Slurries that were only oxidized (1) did not exfoliate $\mathrm{MoS}_{2}$ to any appreciable amount due to lack of POMs to initiate delamination. Similarly, addition of reductant (HQ) only (2) without oxidation results in no exfoliation as there are no MOPs to assemble into POMs. If a full redox cycle is done (oxidation to generate MOPs and reduction to assemble into POMS), however, delamination occurs regardless of reductant used $\left(\mathrm{NaBH}_{4}, \mathrm{HQ}\right)$..

Table S4. Quantification of $\mathrm{MoS}_{2}$ concentrations after each process. Experimentally determined $\mathrm{MoS}_{2}$ concentrations are evaluated 24 hours after addition of reductant. Slurries were centrifuged at $10000 \mathrm{rpm}$ to sediment all $\mathrm{MoS}_{2}$. The supernatant, containing reductant, MOPs, and non-adsorbed POMs was removed, and fresh, anhydrous acetonitrile was added in equi-volume. The slurry was mixed via a vorticer, briefly sonicated (c.a. 30s) and centrifuged at $1500 \mathrm{rpm}$ for 10 minutes to sediment bulk, unexfoliated flakes. The supernatant, containing few to monolayer $\mathrm{MoS}_{2}$ was extracted and assayed via UV-Vis spectroscopy to determine dispersed concentrations. 


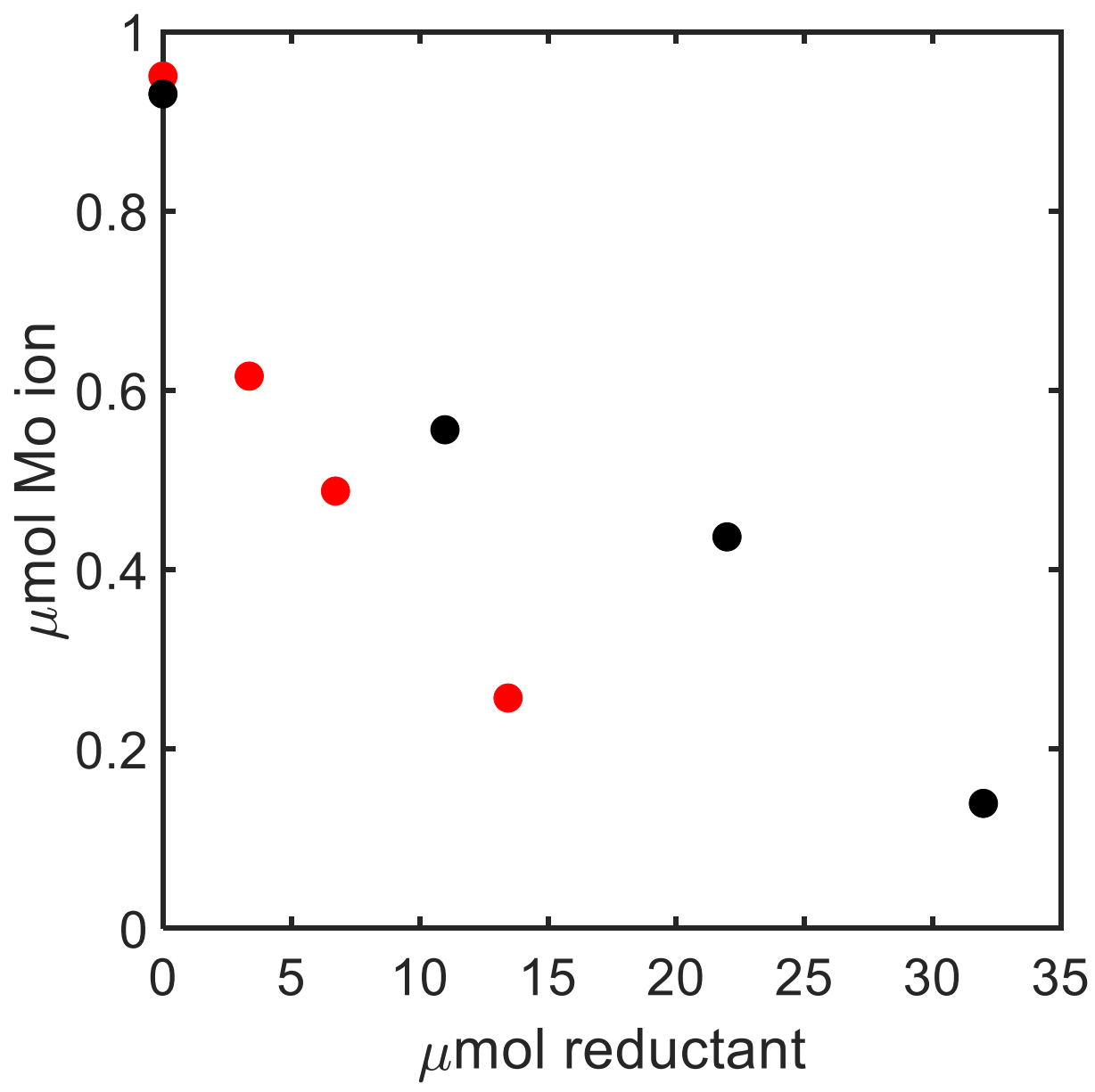

Figure S7. Titration Curves of Mo ions. At a constant Mo ion concentration, the molar efficiency of each reductant determines it's stoichiometric ratio (Red, $\mathrm{NaBH}_{4}$; black hydroquinone) due to the number of electrons available for transfer per molecular unit. 

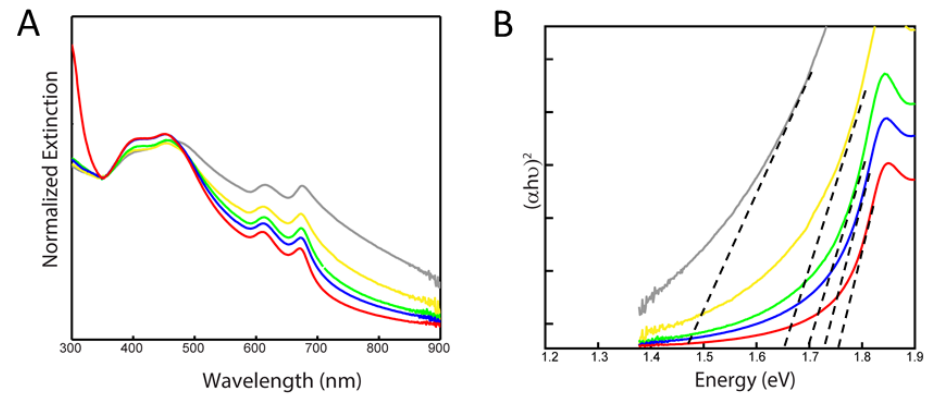

Table S5

\begin{tabular}{|c|c|c|c|}
\hline$\mu \mathrm{mol} \mathrm{HQ}$ & $\begin{array}{c}\lambda_{\mathrm{a}} \\
(\mathrm{nm})\end{array}$ & $\begin{array}{c}\mathrm{E}_{\mathrm{g}} \\
(\mathrm{eV})\end{array}$ & $\begin{array}{c}\text { \# of } \\
\text { layers }\end{array}$ \\
\hline 10 & 680 & 1.46 & 20 \\
\hline 20 & 677 & 1.65 & 14 \\
\hline 30 & 674 & 1.7 & 8 \\
\hline 40 & 672 & 1.72 & 6 \\
\hline 70 & 670 & 1.76 & 4 \\
\hline
\end{tabular}

Figure S8. Delamination of $\mathrm{MoS}_{2}$ via Redox Exfoliation. A) Normalized UV-Vis spectra of $\mathrm{MoS}_{2}$ dispersed in acetonitrile as an increasing amount of hydroquinone is added. As few-to-monolayer flakes disperse, the scattering intensity from bulk-like flakes decreases and A-excitonic features blue shift to direct-gap like transitions B) Tauc plots of exfoliated $\mathrm{MoS}_{2}$ dispersions indicating increasing degree of exfoliation as more reductant is added, approaching the monolayer optical gap of $1.8 \mathrm{eV} .^{3}$

Table S3) Summary of optical gaps, and A-exciton position as a function of addition of reductant. As more POMs are formed, the extent of exfoliation increases, indicated by optical gaps increasing in energy towards the monolayer limit $(1.8 \mathrm{eV})$ and blue-shift of A-excitonic feature. 


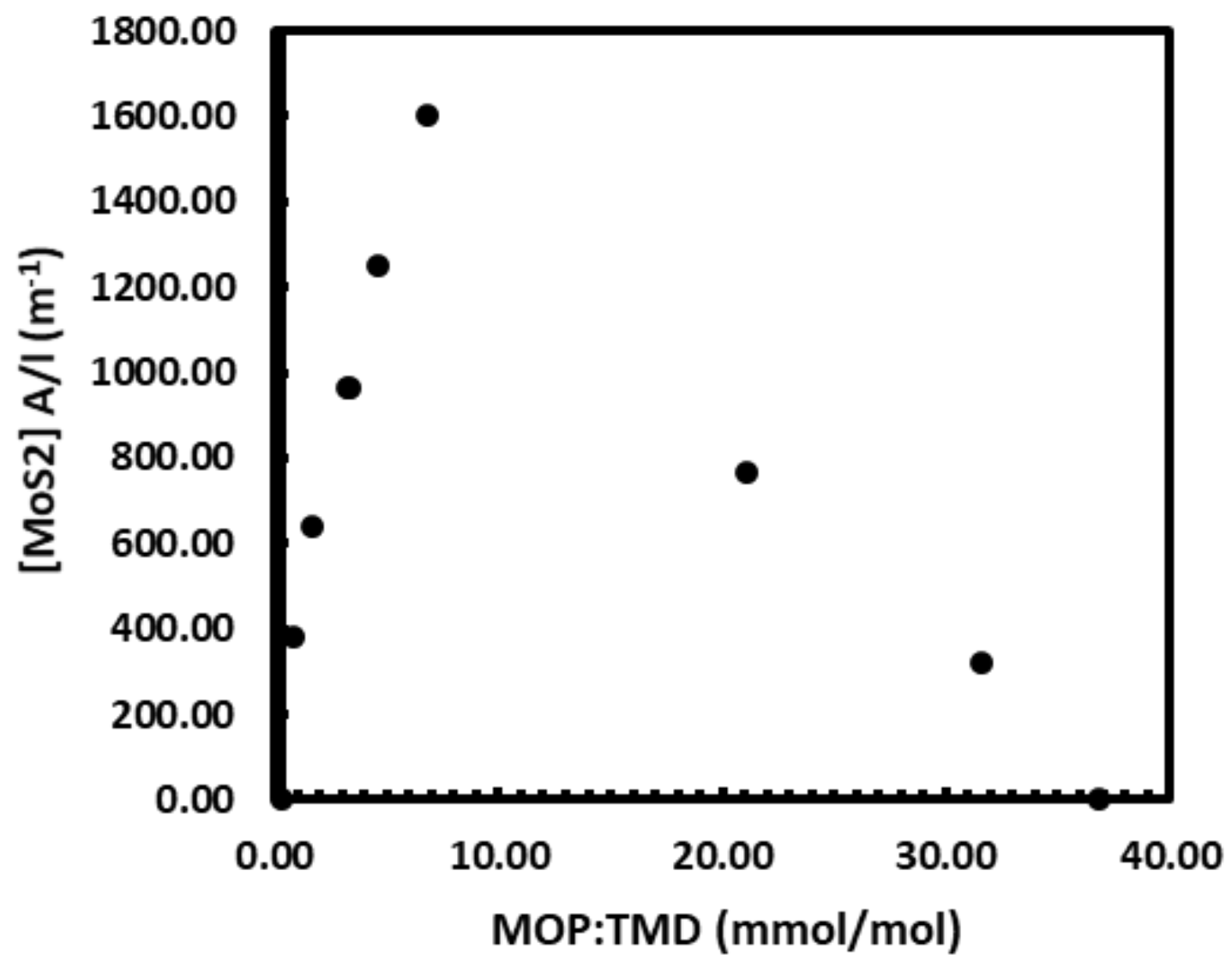

Figure S9 MOP:MoS2 ratio. Very high MOP:TMD ratios result in reduced delamination. This is likely due to ionic screening effects inhibiting charged $\mathrm{MoS}_{2}$ species from entering the liquid phase, which is similar in effect to what has been observed with surfactant assisted exfoliation strategies, where exfoliation yields drop off dramatically beyond the $\mathrm{CMC}$. Because both these methods rely on surface charge stabilization of $\mathrm{MoS}_{2}$, soluble ionic species will create a large enthalpic penalty for dispersing in the liquid phase. Beyond c.a. 30:1 MOP:TMD ratio, the soluble POM charges completely suppress $\mathrm{MoS}_{2}$ delamination. 

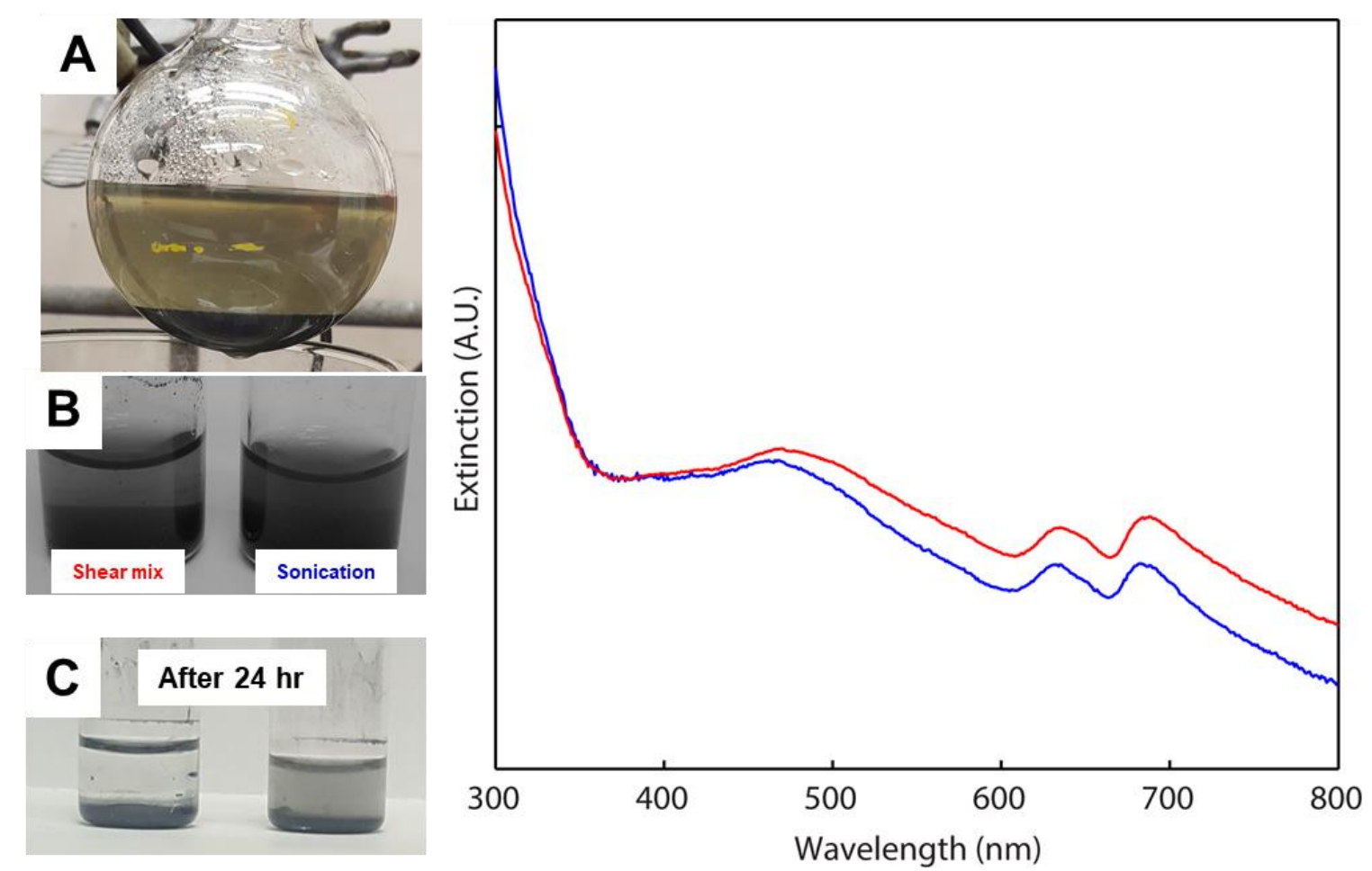

Figure S10. A) $\mathrm{MoS}_{2}$ slurry after oxidation with cumene hydroperoxide in acetonitrile after stirring is stopped. Immediately after the stir is removed, bulk powders sediment, and the supernatant is an optically clear, yellow solution, containing MOPs. B) subjecting the slurry to shear mixing (10k rpm, $1 \mathrm{hr}$ ) or sonication $(1 \mathrm{hr}, 125 \mathrm{~W})$ results in no exfoliation as indicated by the gray suspension of bulk materials in the liquid phase. C) After 24 hours, no MoS 2 is suspended in the liquid phase, indicating poorly colloidally stable bulk crystallites after mechanical agitation. D) UV-Vis spectra of sonicated and shear-mixed slurry. The position of the A-exciton $(684 \mathrm{~nm})$ and large scattering slope are indicative of large bulk-like aggregates and not exfoliated flakes $\left(\mathrm{l}_{\mathrm{A}} 670 \mathrm{~nm}\right)$ 


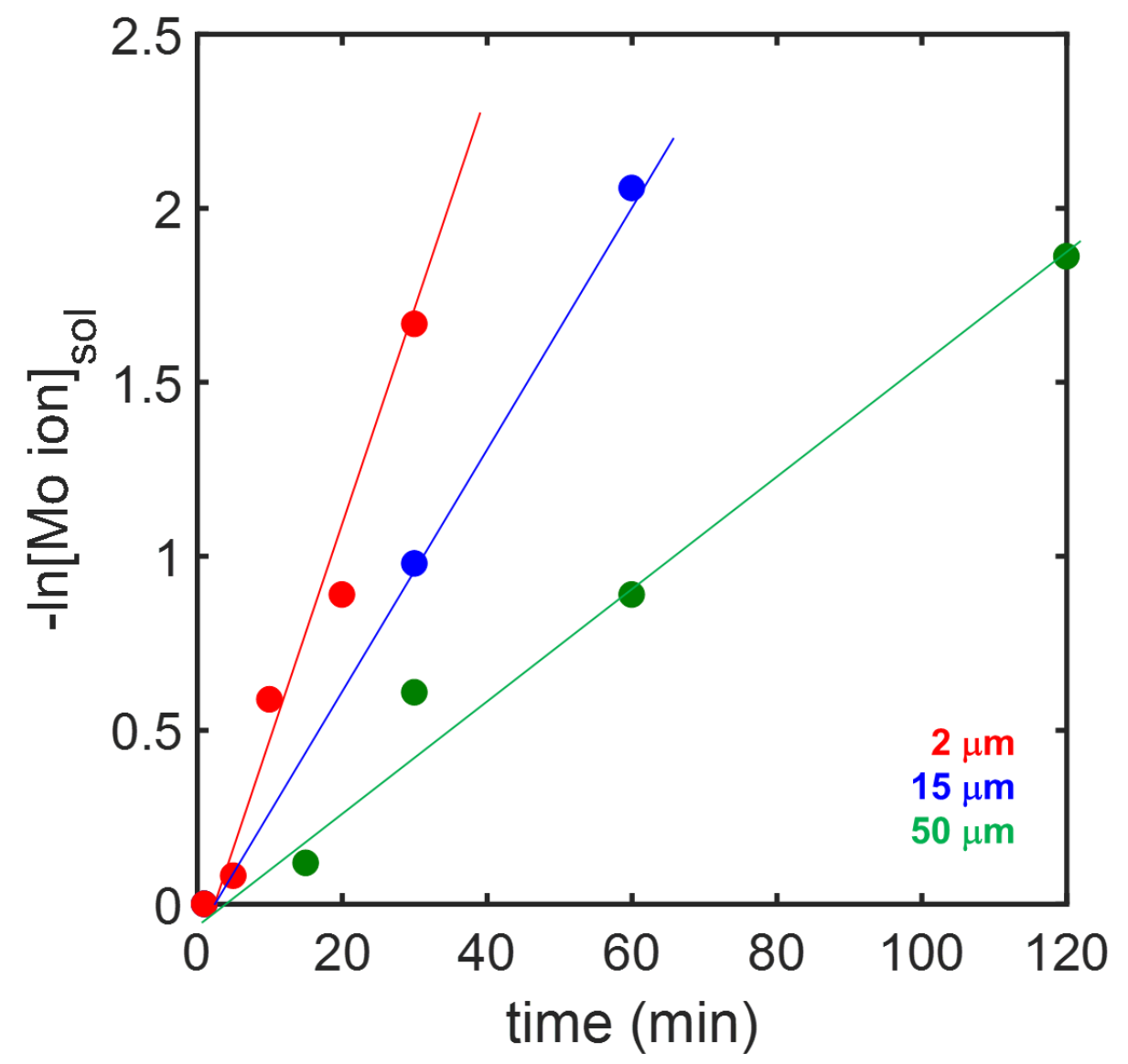

Figure S11. Kinetic plots of Mo ion depletion rates. Depletion rates of Mo ions from solution (after seembly to POMs) indicate a strong correlation with the agglomerate powder size. smaller grains $(2 \mu \mathrm{m})$, having more surface area available, adsorb POMs much more rapidly than coarser powders $(15,50 \mu \mathrm{m})$. Thus, penetration of POMs into larger agglomerates is a surface/edge effect, evidenced by the inverse correlation of powder size and POM adsorption rate. 

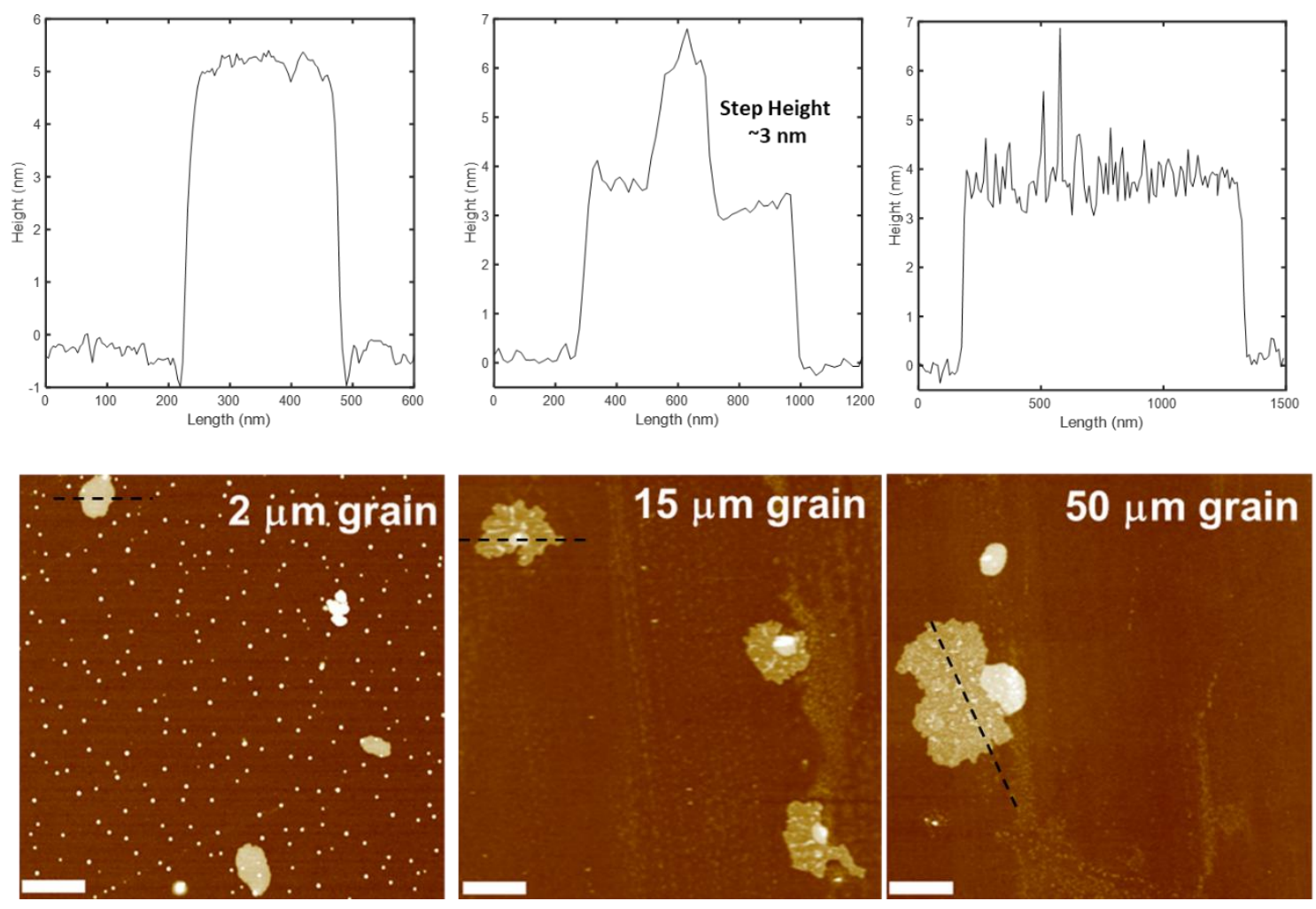

Figure S12 Line Cuts of Exfoliated Flakes. Line cuts of exfoliated flakes with different grain sizes. Observed heights are $3-6 \mathrm{~nm}$, corresponding to $1-2$ layers of MoS2. In accordance with previous literature, the observed monolayer in liquid processed flakes are typically larger than the absolute gap $(0.65 \mathrm{~nm})$ due to solvent effects and surface adsorbates. However, approximate layer content can be determined via analyzing any quantized steps in the flake morphology. For Redox Exfoliated flakes, step sizes observed are typically $3 \mathrm{~nm}$, indicating that flakes produced are $1-2$ layers in thickness. 

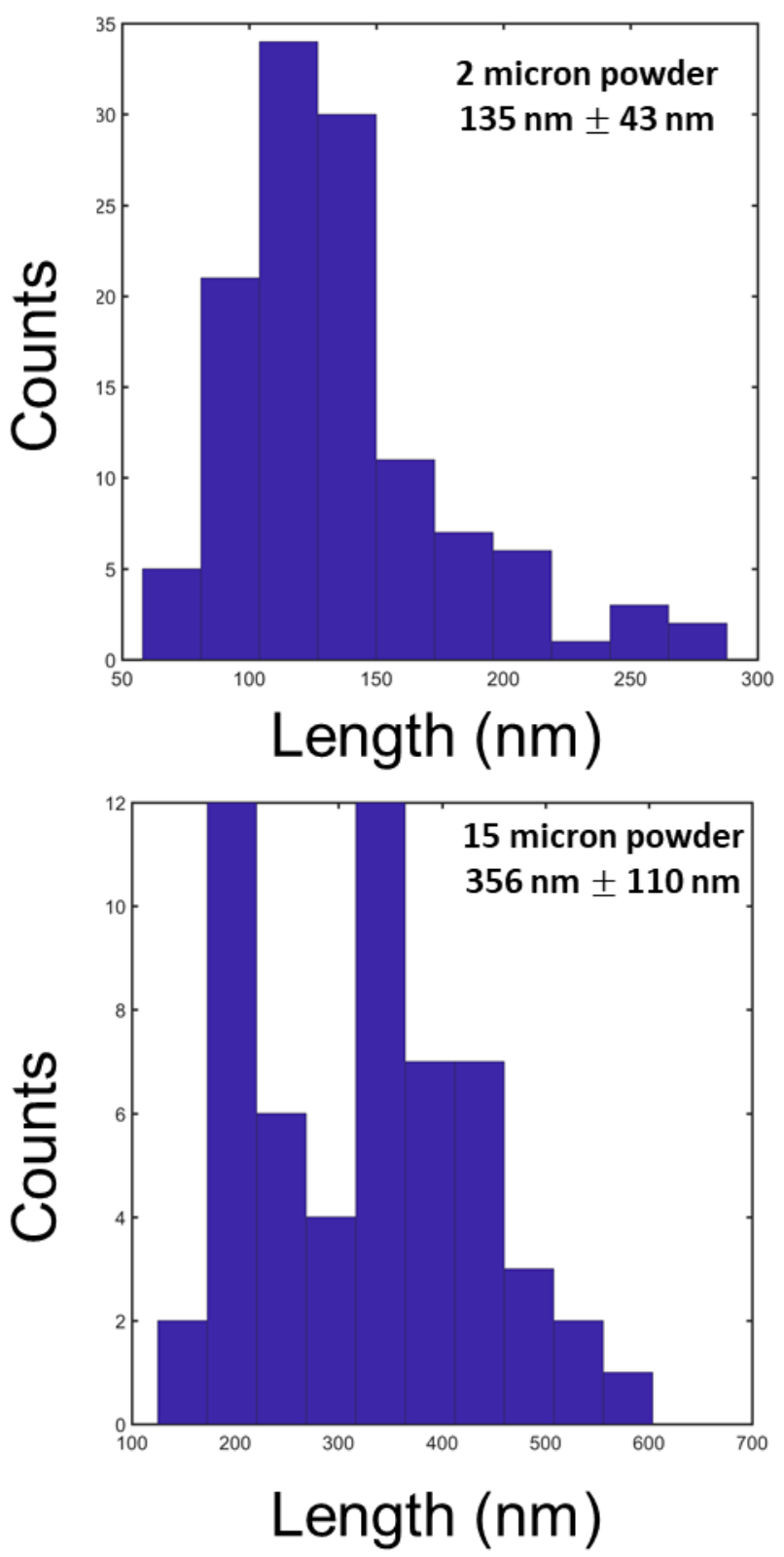

Figure S13. Lateral Size of Exfoliated Flakes. Lateral size analysis of 2 micron and 15 micron powders indicate the average exfoliated flake length varies across suppliers. This is likely due to differences in mean crystallite dimensions. Note, 2 and 15 micron does not represent the average crystallite dimension; rather, it is reflective of the size of the bulk agglomerates. 


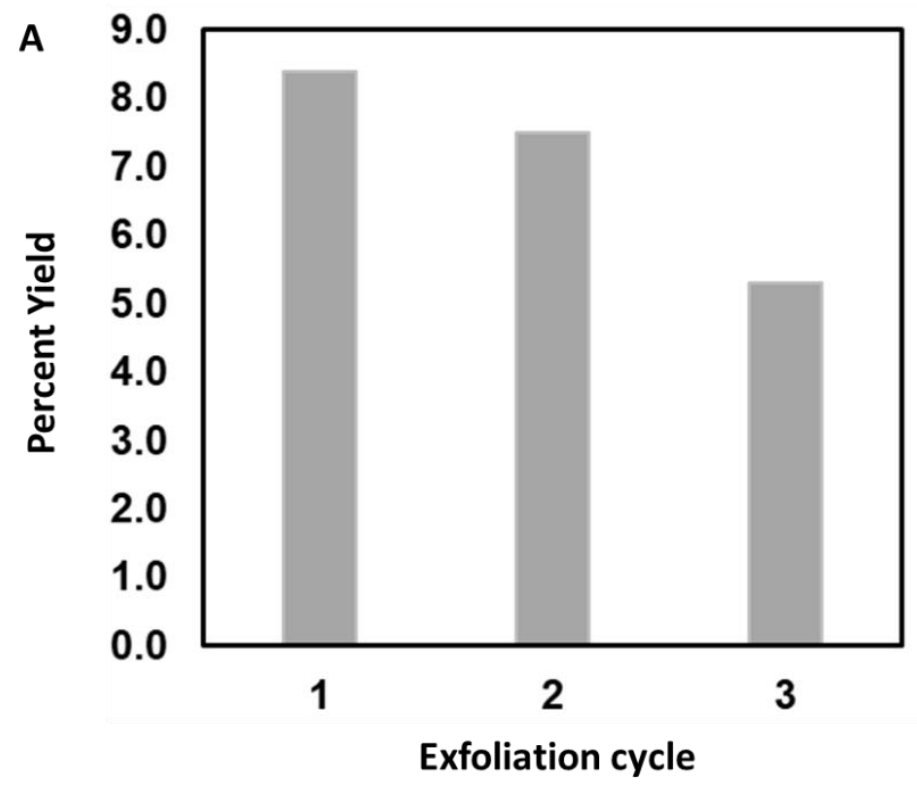

B

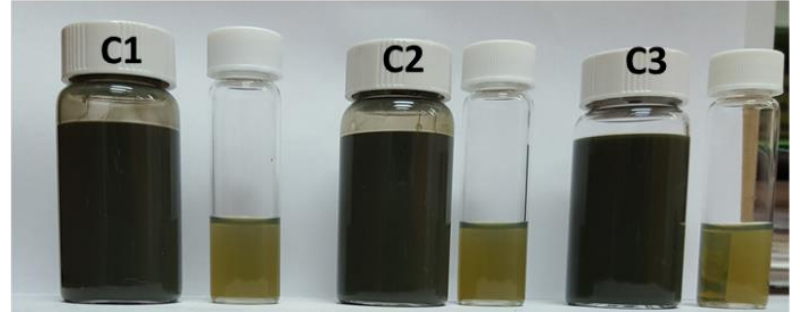

Figure S14. Recycled Exfoliation Reactions. (A) Exfoliation of bulk powders (Sigma, 2 micron) results in $\sim 8 \%$ yield by mass of exfoliated flakes. Extracting un-exfoliated bulk powders, washing, and initiating additional cycles of the Redox Exfoliation process results in upwards of $7 \%$ exfoliation by mass through additional cycles (up to 3 cycles were tested). Initially, $1500 \mathrm{mg}$ of $\mathrm{MoS}_{2}$ was subjected to redox (cycle 1, exfoliated mass 130 $\mathrm{mg} ; 8 \%$ yield). The remaining bulk powder was washed, dried, and subjected to an additional redox cycle (Cycle 2 initial mass $1300 \mathrm{mg}$; exfoliated mass $91 \mathrm{mg}$ ). After the $3^{\text {rd }}$ cycle (initial mass $1150 \mathrm{mg}$; exfoliated mass $80 \mathrm{mg}$ ), a cumulative yield for an initial $1500 \mathrm{mg}$ of powder was $20 \%$ of exfoliated $\mathrm{MoS}_{2}$ by mass. (B) Optical image of $\mathrm{MoS}_{2}$ exfoliated after each redox cycle. $\mathrm{C}_{1}:\left[\mathrm{MoS}_{2}\right]=8.1 \mathrm{mg} / \mathrm{mL} ; 16 \mathrm{~mL}$. C2: $\left[\mathrm{MoS}_{2}\right]=5.5$ $\mathrm{mg} / \mathrm{mL}, 16 \mathrm{~mL}$; 3 : $\left[\mathrm{MoS}_{2}\right]=5.1 \mathrm{mg} / \mathrm{mL} ; 16 \mathrm{~mL}$. Optically transparent vials are diluted 300 fold. 


\section{References.}

1. Griffin, A. et al. Effect of Surfactant Choice and Concentration on the Dimensions and Yield of Liquid-Phase-Exfoliated Nanosheets. Chem. Mater. (2020)

2. Jawaid, A. et al. Mechanism for Liquid Phase Exfoliation of MoS2. Chemistry of Materials 28, 337-348 (2016).

3. Mak, K. F., Lee, C., Hone, J., Shan, J. \& Heinz, T. F. Atomically Thin MoS2: A New Direct-Gap Semiconductor. Phys. Rev. Lett. 105, 136805 (2010). 